\title{
FDISCO+: a clearing method for robust fluorescence preservation of cleared samples
}

\author{
Peng Wan, ${ }^{\text {a,b }}$ Yusha Li, ${ }^{\text {a,b }}$ Jingtan Zhu, ${ }^{\text {a,b }}$ Jianyi Xu, ${ }^{\text {a,b }}$ Xiaomei Liu, \\ Tingting $\mathbf{Y u},{ }^{\mathrm{a}, \mathrm{b}, *}$ and Dan Zhu $\oplus^{\mathrm{a}, \mathrm{b}}$ \\ ${ }^{a}$ Huazhong University of Science and Technology, Britton Chance Center for \\ Biomedical Photonics, Wuhan National Laboratory for Optoelectronics, Wuhan, China \\ ${ }^{b}$ Huazhong University of Science and Technology, MoE Key Laboratory for Biomedical \\ Photonics, School of Engineering Sciences, Wuhan, China
}

\begin{abstract}
Significance: The recently reported solvent-based optical clearing method FDISCO can preserve various fluorescent signals very well. However, the strict low-temperature storage condition of FDISCO is not conducive to long-time or repetitive imaging usually conducted at room temperature. Therefore, it is important to solve the contradiction between fluorescence preservation and imaging condition.

Aim: We develop a modified FDISCO clearing method, termed FDISCO+, to change the preservation condition from low temperature to room temperature.

Approach: Two alternative antioxidants were screened out to effectively inhibit the peroxide generation in the clearing agent at room temperature, enabling robust fluorescence preservation of cleared samples.

Results: FDISCO+ achieves comparable fluorescence preservation with the original FDISCO protocol and allows long-time storage at room temperature, making it easier for researchers to image and preserve the samples.

Conclusions: FDISCO+ is expected to be widely used due to its loose operation requirements.

(C) The Authors. Published by SPIE under a Creative Commons Attribution 4.0 Unported License. Distribution or reproduction of this work in whole or in part requires full attribution of the original publication, including its DOI. [DOI: 10.1117/1.NPh.8.3.035007]
\end{abstract}

Keywords: optical clearing; FDISCO+; fluorescence preservation; room temperature; fluorescence imaging; antioxidant.

Paper 21021R received May 10, 2021; accepted for publication Aug. 24, 2021; published online Sep. 9, 2021.

\section{Introduction}

It has always been a goal of biologists to obtain three-dimensional (3D) structures of large tissues and organs at high resolution, especially when it comes to reconstructing complex networks, like neural and vascular systems. ${ }^{1-5}$ Recently, the technique of "optical sectioning" provides a powerful tool for repetitive and efficient 3D tissue imaging. ${ }^{6-8}$ However, due to the cloudy features of biological tissue, imaging depth is limited among numerous optical fluorescence microscopes such as the confocal microscope, multi-photon microscope, and light-sheet microscope. ${ }^{1,9-12}$ In recent years, this problem has been partially solved thanks to the rapid development of optical clearing methods, which can reduce the light scattering and light absorption in the tissues to achieve deep imaging in combination with optical fluorescence microscopy. ${ }^{13,14}$

Over the years, a variety of optical clearing methods have been developed..$^{15,16}$ They are usually divided into three categories: the hydrophobic clearing methods, such as $\mathrm{BABB},{ }^{17}$ 3 DISCO,${ }^{18-20}$ iDISCO,${ }^{21}$ uDISCO,${ }^{22}$ FluoClearBABB,${ }^{23}$ Ethanol-ECi, ${ }^{24}$ vDISCO,${ }^{25}$ sDISCO,${ }^{26}$

*Address all correspondence to Tingting Yu, yutingting@hust.edu.cn 
a-uDISCO,${ }^{27}$ PEGASOS,${ }^{28}$ and FDISCO $;{ }^{29}$ the hydrophilic clearing methods, such as ScaleS,${ }^{30}$ SeeDB,${ }^{31,32}$ Clear ${ }^{T},{ }^{33}$ CUBIC $,{ }^{34-36} \mathrm{C}_{\mathrm{e}} 3 \mathrm{D},{ }^{37} \mathrm{RTF}^{38}$ and MACS $;{ }^{5}$ and the hydrogel-based clearing methods, such as CLARITY ${ }^{39,40}$ and PACT-PARS. ${ }^{41-43}$ Different clearing methods are usually applied for certain scopes, and they have respective strengths and weaknesses: the aqueousbased clearing methods usually show good fluorescence preservation, and the organic solventbased clearing methods usually have excellent clearing capability. ${ }^{44}$

The solvent-based clearing methods are widely used due to their characteristics of reduced sample size and high transparency, as size reduction can break the limit of working distance of objective to image larger volumes, and high transparency allows imaging deeper. ${ }^{4,22} 3 \mathrm{DISCO}$, as an early published method, has advantages on both short clearing time and high tissue transparency. However, 3DISCO is not compatible with endogenous fluorescent molecules. ${ }^{19,22}$ To address this issue, FDISCO was recently proposed by modifying the experimental conditions of 3DISCO and realizes the preservation of the endogenous fluorescence signals, expanding the application scope of DISCO methods. ${ }^{29}$ However, FDISCO requires a strict storage condition that the cleared samples must be stored at low temperature (LT) $\left(4^{\circ} \mathrm{C}\right.$ to $\left.8^{\circ} \mathrm{C}\right)$ because the fluorescence signals would be rapidly and severely quenched at room temperature (RT), whereas the imaging apparatus of conventional fluorescence microscopy is generally under RT environment during imaging. As a compromise between the fluorescence preservation and the imaging temperature, the FDISCO-cleared samples must be imaged in a short time. But contradictions are inevitable when it comes to long-time or repetitive imaging operation. In this case, the fluorescence would decrease; hence imaging at RT might influence the imaging quality and the visualization of tissue structures.

In this study, we developed FDISCO+, a clearing method that optimizes the preservation condition of FDISCO from LT to RT. The FDISCO+ achieves comparable fluorescence enhancement with FDISCO and preserves the endogenous fluorescence at RT for a long time, making it easier for researchers to image and store the samples. Due to its loose operation requirements, FDISCO+ is expected to be widely used.

\section{Methods}

\subsection{Animals}

In this study, we used Thyl-GFP-M mice, CX3CRl-GFP mice, and C57BL/6J mice (6 to 12 weeks old). Mice were anesthetized with a mixture of $2 \% \alpha$-chloralose and $10 \%$ urethane $(0.8 \mathrm{~mL} / 100 \mathrm{~g})$ via intraperitoneal injection. Next, mice were transcardially perfused with $0.01 \mathrm{M}$ phosphate-buffered saline (PBS) (P3813, Sigma-Aldrich) and then 4\% paraformaldehyde (PFA) (158127, Sigma-Aldrich). Finally, the brains were dissected and postfixed overnight at $4^{\circ} \mathrm{C}$ in $4 \%$ PFA. Some brains were sliced into $1-\mathrm{mm}$-thick or 2 -mm-thick coronal sections with a vibratome (Leica VT 1200s). The animal care and experimental protocols were in accordance with the Experimental Animal Management Ordinance of Hubei Province, China and have been approved by the Institutional Animal Ethics Committee of Huazhong University of Science and Technology.

\subsection{Chemicals and Reagents}

In this study, we used the following chemicals: tetrahydrofuran (THF) (186562, Sigma-Aldrich), dibenzyl ether (DBE) (108014, Sigma-Aldrich), $N, N, N^{\prime}, N^{\prime}$-tetrakis (2-hydroxypropyl) ethylenediamine (EDTP) (T0781, Tokyo Chemical Industry Co., Ltd.), DL-alpha-tocopherol (vitamin E) (A17039, Alfa Aesar), and triethylamine (80134318, Sinopharm Chemical Reagent Co. Ltd.).

For dehydration solutions, THF was mixed with $\mathrm{dH}_{2} \mathrm{O}$ according to the following concentration: $50 \%(\mathrm{v} / \mathrm{v}), 70 \%(\mathrm{v} / \mathrm{v}), 80 \%(\mathrm{v} / \mathrm{v})$, and $100 \%(\mathrm{v} / \mathrm{v})$. Triethylamine was added to adjust the $\mathrm{pH}$ to about 9.0. For clearing agents, the eFDISCO+ was prepared by adding $0.5 \%(\mathrm{w} / \mathrm{v})$ EDTP to the DBE, and the vFDISCO+ was prepared by adding $0.5 \%(\mathrm{v} / \mathrm{v})$ vitamin $\mathrm{E}$ to the DBE. 
The column absorption chromatography with basic activated aluminum oxide (20001861, Sinopharm Chemical Reagent Co. Ltd.) was used to remove the peroxides produced in THF and DBE, as described in the literature. ${ }^{19,20}$

\subsection{Measurement of Peroxide Concentration}

The basic activated aluminum oxide was used to remove the peroxides in DBE. Then EDTP at concentrations of $0.1 \%(\mathrm{w} / \mathrm{v}), 0.5 \%(\mathrm{w} / \mathrm{v}), 1 \%(\mathrm{w} / \mathrm{v})$, and $2 \%(\mathrm{w} / \mathrm{v})$; vitamin $\mathrm{E}$ at concentrations of $0.1 \%(\mathrm{v} / \mathrm{v}), 0.5 \%(\mathrm{v} / \mathrm{v}), 1 \%(\mathrm{v} / \mathrm{v})$, and $2 \%(\mathrm{v} / \mathrm{v})$; and triethylamine at concentrations of $0.1 \%$ $(\mathrm{v} / \mathrm{v}), 0.5 \%(\mathrm{v} / \mathrm{v}), 1 \%(\mathrm{v} / \mathrm{v})$, and $2 \%(\mathrm{v} / \mathrm{v})$ were added to $10 \mathrm{~mL} \mathrm{DBE}$ in $50 \mathrm{~mL}$ centrifugal tubes (430829, Corning, USA) to prepare different refractive index (RI) matching solutions, respectively. Quantofix-25 strips (Macherey-Nagel, Germany) were used to measure the peroxide concentration of the reagent after $0,9,24$, and $72 \mathrm{~h}$. All tubes except the pure DBE group at LT were placed horizontally in a dark room at RT. The pure DBE group at LT was placed in a refrigerator at $4^{\circ} \mathrm{C}$ to $8^{\circ} \mathrm{C}$.

\subsection{FDISCO+ Clearing Procedure}

The 1-mm-thick brain sections were dehydrated with THF solutions at graded concentrations: $50 \%(\mathrm{v} / \mathrm{v}), 70 \%(\mathrm{v} / \mathrm{v}), 80 \%(\mathrm{v} / \mathrm{v})$, and $100 \%(\mathrm{v} / \mathrm{v})(\mathrm{twice})$, with $1 \mathrm{~h}$ each step at $4^{\circ} \mathrm{C}$ to $8^{\circ} \mathrm{C}$. Then the dehydrated samples were transferred to the RI matching solutions for 30 min at RT.

For whole-brain clearing, the samples were dehydrated with $50 \%(\mathrm{v} / \mathrm{v}), 70 \%(\mathrm{v} / \mathrm{v}), 80 \%(\mathrm{v} / \mathrm{v})$, and $100 \%(\mathrm{v} / \mathrm{v})\left(\right.$ thrice) THF solutions, with $12 \mathrm{~h}$ each step at $4{ }^{\circ} \mathrm{C}$ to $8^{\circ} \mathrm{C}$, followed by immersing in the RI matching agents for more than $3 \mathrm{~h}$ at RT.

During the clearing, the samples were covered with aluminum foil to ensure a dark environment, and all steps were performed with slight shaking. The cleared samples were stored at RT all the time.

\subsection{Fluorescence Labeling}

For nuclei staining, the 1-mm-thick brain sections were incubated with $1 \%(\mathrm{w} / \mathrm{v})$ propidium iodide (PI) (P1304MP, Life Technologies)/PBST (0.2\% Triton X-100/PBS) for $24 \mathrm{~h}$ at RT with slight shaking. Then sections were washed several times with PBST for $6 \mathrm{~h}$.

For vasculature labeling, $10 \mu \mathrm{g}$ Alexa Fluor 647 conjugated anti-mouse CD31 antibody (CD31-A647) (102416, BioLegend) was used to label the vasculature in C57BL/6J mouse brain by caudal vein injection. The mouse was perfused $30 \mathrm{~min}$ after injection. The postfixed mouse brain was sliced into 1-mm-thick coronal sections.

\subsection{Measurement of Transparency}

A digital camera (HDC-HS900GK) was used to acquire the bright-field images of samples. We used a visible-near-infrared optical fiber spectrometer (USB4000, Ocean Optics, USA) to produce a circular spot of light (diameter, $5 \mathrm{~mm}$ ) to irradiate the samples immersed in the cleared medium. Then the transmitted light was measured on the other side of the samples. The light across only the clearing medium was measured as the blank value. Finally, the light transmittance was normalized by the blank value.

\subsection{Fluorescence Imaging}

For brain slices, the cortical regions of the samples were imaged with a confocal fluorescence microscope (LSM 710, Zeiss, Germany) equipped with objectives of Fluar $5 \times / 0.25$ (dry; working distance, $12.5 \mathrm{~mm}$ ) and Fluar $10 \times / 0.5$ (dry; working distance, $2.0 \mathrm{~mm}$ ). Before and after clearing, the imaging parameters were kept the same when imaging the same regions.

For whole brains, samples were imaged with a customized Bessel light-sheet fluorescence microscope (LSFM) based on Olympus MVX10 and equipped with an sCMOS camera (Hamamatus Flash $4.0 \mathrm{~V} 2$ ), as described in the published literature. ${ }^{45}$ In this study, $3 \mathrm{~h}$ to $4 \mathrm{~h}$ are needed for imaging per whole mouse brain. 


\subsection{Data Analysis}

The Fiji (Version 1.51n), MATLAB (Version 2014a, MathWorks), and Imaris (Version 7.6, Bitplane AG) were used for image processing and quantitative analysis of data. The analysis was derived from the literature. ${ }^{44,46}$

For the quantification of GFP fluorescence in CX3CR1-GFP mouse brain, the maximum intensity projections (MIPs) of $25 \mu \mathrm{m}$ thickness were acquired from each $z$ stack. We used Otsu algorithm for image thresholding segmentation and binarization. ${ }^{47}$ Then we used MATLAB to extract the signal value and calculate the mean fluorescence intensity of the MIP images. The normalized fluorescence intensity was calculated by dividing the mean fluorescence intensity after $n(n=3,6,9) \mathrm{h}$ by the value after $0 \mathrm{~h}$. The processing is similar for quantification of PI signals, CD31-A647 signals, and GFP fluorescence for long-time preservation. And the normalized value of PI and CD31-A647 signals was described as the ratio of the mean fluorescence intensity before and after clearing, and the normalized GFP fluorescence intensity for long-time preservation was calculated by dividing the mean fluorescence intensity after $n(n=3,7,14)$ days by the mean fluorescence intensity after 0 day.

For the quantification of GFP fluorescence enhancement in Thyl-GFP-M mouse brain, we used Fiji to outline the same neurons in the cortical areas before and after clearing and measure the mean fluorescence intensity. The selective neurons must be located in the shallow position to avoid the influence of light scattering on the fluorescence intensity. The normalized mean fluorescence intensity was described by dividing the intensity of cleared group by the value of the uncleared group.

For the LSFM images, we used Fiji to transform the data to 8-bit images and then stitched the images using the Fiji plugins of Grid/Collection Stitching. Finally, we used Imaris to visualize the 3D-rendered images.

\section{Results}

\subsection{Development of FDISCO+ by Inhibiting the Generation of Peroxides in DBE}

The peroxide contaminations generated in DBE would quench the endogenous fluorescence severely even at a low concentration. ${ }^{19}$ FDISCO realizes the good preservation of fluorescence in DBE by controlling the temperatures at a low level. ${ }^{29}$ In this work, we measured the peroxide concentration in DBE using Quantofix-25 strips. As shown in Figs. 1(a) and 1(b), the DBE at RT produced about $0.5 \mathrm{mg} / \mathrm{L}$ peroxides in $9 \mathrm{~h}$, whereas the DBE at $\mathrm{LT}$ did not produce peroxides in $72 \mathrm{~h}$. This result indicates that LT can effectively reduce the generation of peroxides in DBE, facilitating fluorescence preservation. Hence, the samples cleared by FDISCO have to be kept at LT all the time to minimize fluorescence loss of fluorescence intensity for follow-up imaging, especially for the samples that needed repetitive imaging. However, it is not easy to achieve LT during imaging, as the imaging apparatus is usually under RT environment.

To solve the conflict between fluorescence preservation and imaging temperature, we look for a modified protocol based on FDISCO to preserve endogenous fluorescence well at RT. First, we chose three reagents, including EDTP, vitamin E, and triethylamine and tested their antiperoxidation capability when added to DBE. As shown in Figs. 1(a) and 1(b), DBE added with EDTP at concentrations higher than $0.5 \%(\mathrm{w} / \mathrm{v}$ ) did not produce peroxides for $72 \mathrm{~h}$, indicating effective inhibition of the peroxide generation in DBE at RT. Additionally, for vitamin E, the concentration of $0.5 \%(\mathrm{v} / \mathrm{v})$ in DBE also did not produce observable peroxides for $72 \mathrm{~h}$. In contrast, DBE added with triethylamine at concentrations of $0.1 \%(\mathrm{v} / \mathrm{v}), 0.5 \%(\mathrm{v} / \mathrm{v})$, $1 \%(\mathrm{v} / \mathrm{v})$, and $2 \%(\mathrm{v} / \mathrm{v})$ induced a faster generation of peroxides than the pure DBE group at RT, indicating that triethylamine could not prevent but accelerate the peroxide generation. These results indicate that the addition of proper concentration of EDTP and vitamin E has antiperoxidation capability on DBE. Further, we validated the influence of the addition of different concentrations of EDTP and vitamin E in DBE on tissue transparency. As shown in Fig. 1(c), the tissue transparency reduced slightly as the concentration of EDTP increased but was not affected by vitamin $\mathrm{E}$ with different concentrations. Considering both the oxidation resistance 


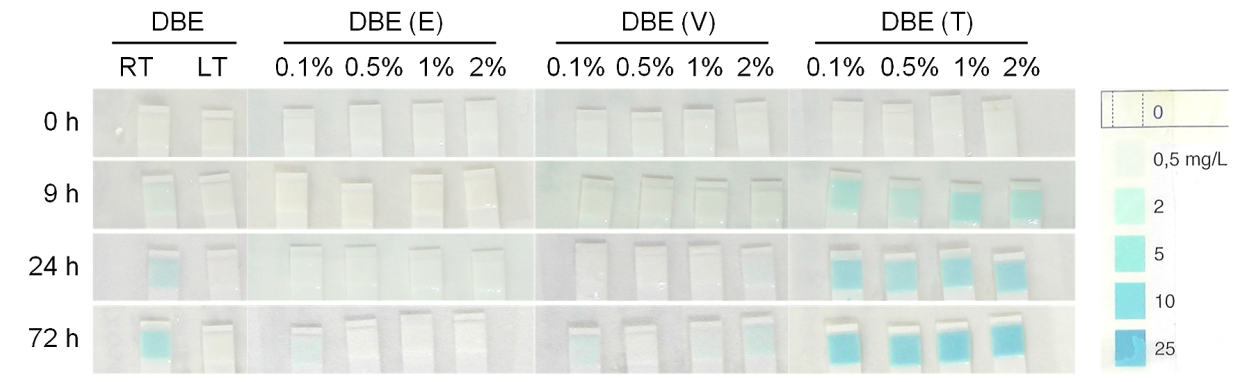

(a)
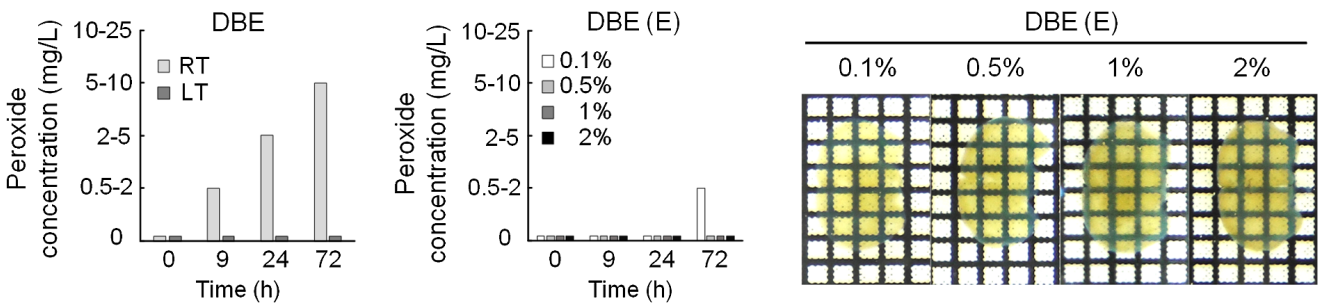
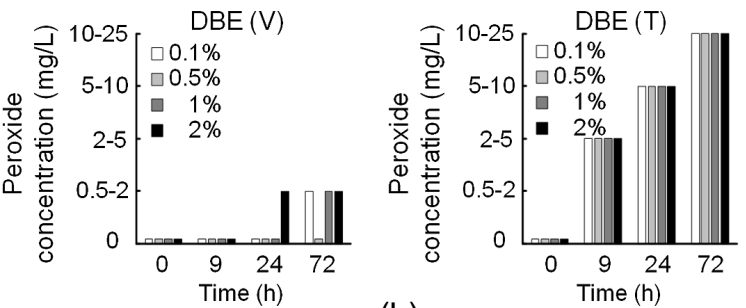

(b)

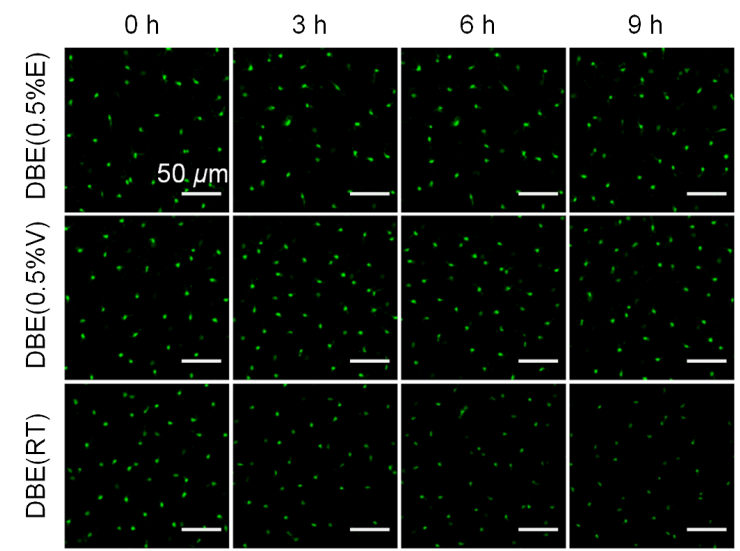

(d)

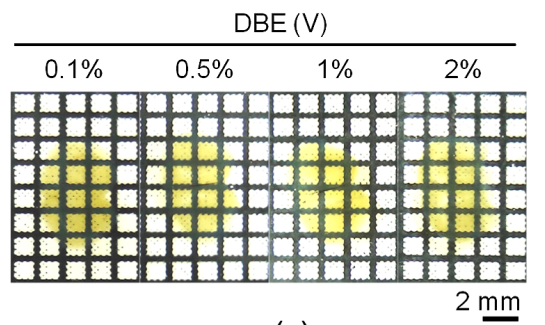

(c)

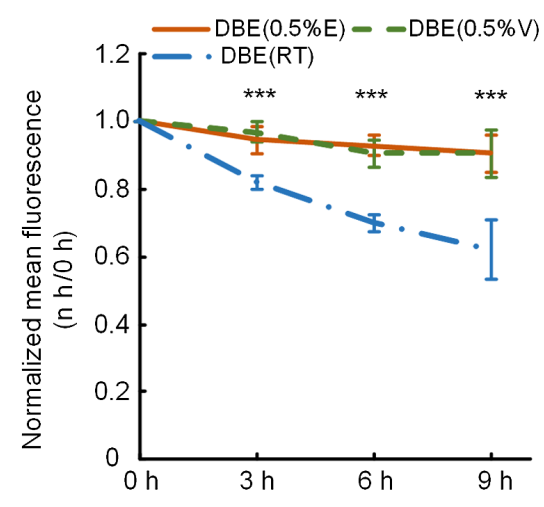

(e)

Fig. 1 Inhibiting the peroxide generation in DBE at RT preserves the GFP fluorescence. (a) The concentration of peroxides in DBE in different experimental conditions at $0,9,24$, and $72 \mathrm{~h}$, respectively. RT: the pure DBE was stored at RT; $L T$ : the pure DBE was stored at $L T$; DBE (E): the DBE was added with EDTP and stored at RT; DBE (V): the DBE was added with vitamin $\mathrm{E}$ and stored at $\mathrm{RT}$; and DBE (T): the DBE was added with triethylamine and stored at RT. (b) The quantification of peroxide concentration in (a). (c) 2-mm-thick C57BL/6J mouse brains were cleared in DBE with different concentrations of EDTP or vitamin E. (d) The cleared adult $C X 3 C R 1$-GFP mouse brain slices were put in RI matching solutions at RT and were imaged at $0,3,6$, and $9 \mathrm{~h}$ with the confocal microscope. (e) The decay curves of fluorescence intensity in (d) $(n=8)$. All values are presented as the mean \pm SD. Statistical significance in $(e)\left({ }^{\star \star \star} p<0.001\right)$ was assessed by one-way ANOVA followed by the Bonferroni post hoc test. 
and the clearing transparency, $0.5 \%(\mathrm{w} / \mathrm{v})$ of EDTP or $0.5 \%(\mathrm{v} / \mathrm{v})$ of vitamin $\mathrm{E}$ was chosen as the final optimal concentration.

Then we used the cleared mouse brain slices (CX3CRI-GFP) to verify the fluorescence preserving capability of the DBE added with $0.5 \%(\mathrm{w} / \mathrm{v})$ EDTP or $0.5 \%(\mathrm{v} / \mathrm{v})$ vitamin E. The cleared samples were immersed in respective clearing agents at RT and imaged every $3 \mathrm{~h}$. For the samples immersed in pure DBE, the fluorescence signal declined to $62 \%$ after $9 \mathrm{~h}$. Although for the samples immersed in the DBE added with EDTP or vitamin E, the GFP signals were significant higher than in pure DBE and showed no noticeable decrease along with time due to inhibited peroxide generation, as shown in Figs. 1(d) and 1(e).

Based on the above results, we proposed an optimized clearing protocol for better fluorescence preservation under RT, termed FDISCO+. We devised two schemes: one using DBE containing $0.5 \%(\mathrm{w} / \mathrm{v})$ EDTP as the clearing medium termed eFDISCO+, and the other using DBE containing $0.5 \%(\mathrm{v} / \mathrm{v})$ vitamin E termed vFDISCO+.

\subsection{FDISCO+ Achieves Comparable Fluorescence Enhancement with FDISCO}

To further investigate the fluorescence preserving capability of FDISCO+, we cleared the mouse brain samples labeled with different fluorescent probes. We imaged the same cortical areas of 1-mm-thick brain sections before and after clearing by FDISCO+ and FDISCO and quantified the intensity change of fluorescence signals. FDISCO+ showed excellent preservation for all tested fluorescence signals, including signals from endogenous GFP, PI, and CD31-A647, which revealed no obvious difference compared to the original FDISCO. The GFP fluorescence intensity of samples cleared by FDISCO+ increases to $175 \%$ to $185 \%$ compared to the fluorescence intensity before clearing, as shown in Figs. 2(a) and 2(b). The fluorescence intensity of PI signals after clearing increases to at least 210\%, as shown in Figs. 2(c) and 2(d). In addition, FDISCO+ also significantly strengthens the CD31-A647 signals, as shown in Figs. 2(e) and 2(f).

\subsection{FDISCO+ Allows Long-Time Preservation of GFP Fluorescence at RT}

Further, we tested the ability of long-time fluorescence preservation of FDISCO+. We imaged the cortical neurons in Thyl-GFP-M mouse brains cleared by FDISCO+ and FDISCO at 0, 3, 7, and 14 days then quantified the fluorescence intensity changes normalized to 0 day.

Figure 3 shows the representative images and the quantitative data of intensity changes. The GFP fluorescence of brain blocks treated by FDISCO+ and FDISCO are preserved very well for at least two weeks compared to the pure DBE (RT) group. eFDISCO+ shows similar fluorescence preservation with FDISCO and better fluorescence preservation than vFDISCO+ after 7 days with a significant difference, indicating that eFDISCO+ is better in long-time fluorescence preservation than vFDISCO+.

\subsection{FDISCO+ Shows Good Clearing Capability and Enables Visualization of the Neural Structures}

Finally, we tested the clearing capability of FDISCO+ and displayed 3D visualization of the nervous system with LSFM imaging. Figures 4(a) and 4(b) show the bright-field images of whole brains before and after FDISCO+ and FDISCO clearing and the transmittance curves with wavelength. vFDISCO+ achieves similar transparency as FDISCO, and eFDISCO+ displays modest clearing ability for the mouse brain. Meanwhile, we imaged the Thyl-GFP-M mouse brain cleared by vFDISCO+ and performed 3D reconstruction from different views, as shown in Figs. 4(c) and 4(d) and Video 1. The fine neuronal structures in different brain regions, such as the cortex, caudate putamen, hippocampus, and midbrain, can be detected effectively, as shown in Figs. 4(e)-4(h). We also obtained the 3D reconstruction of neurons in half brain cleared by eFDISCO+, as shown in Video 2 . 

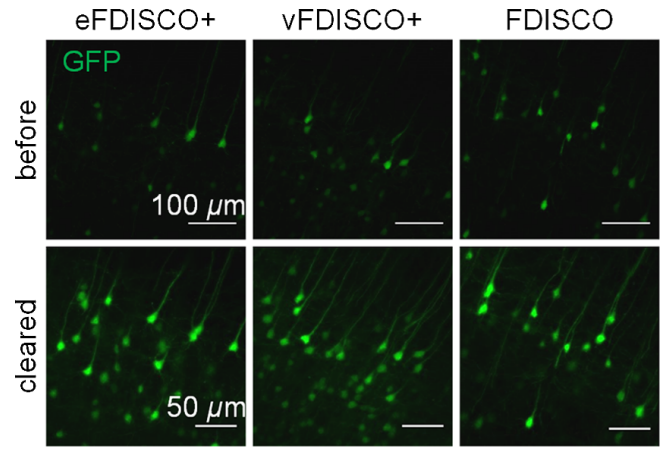

(a)
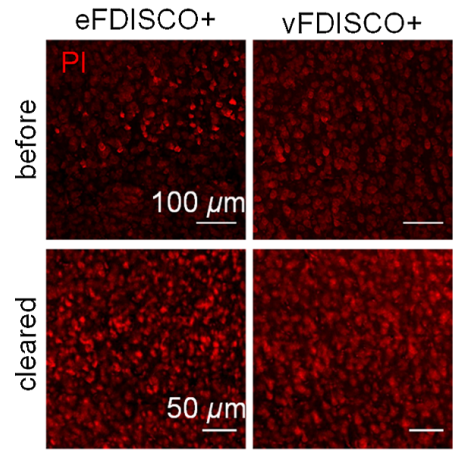

(c)
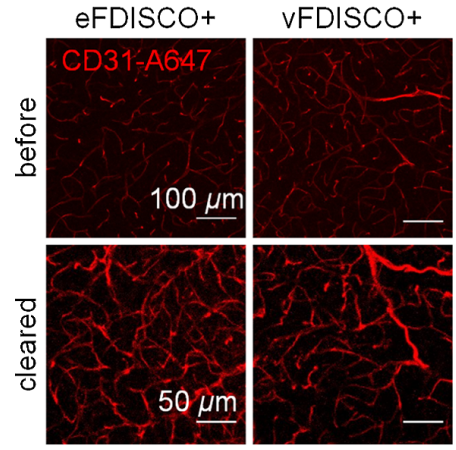

(e)

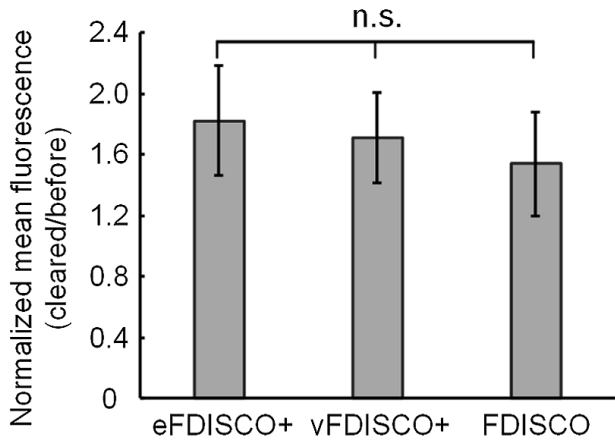

(b)

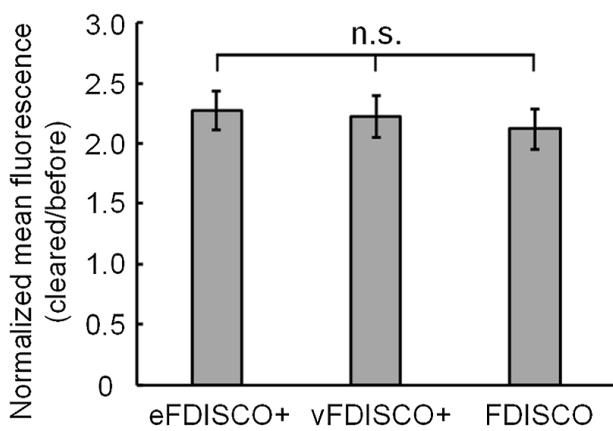

(d)

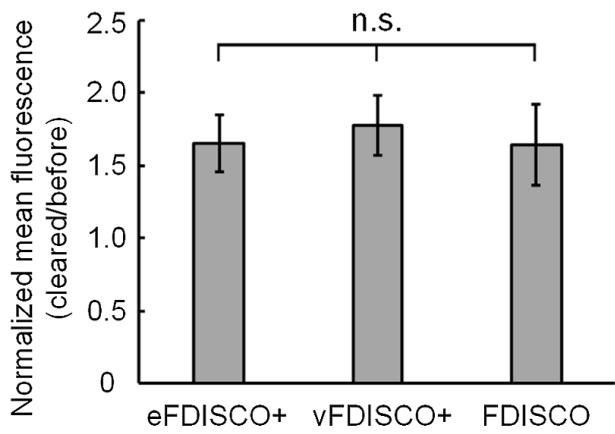

(f)

Fig. 2 FDISCO+ achieves comparable fluorescence enhancement with FDISCO. (a) Confocal imaging of cortical neurons in adult Thy1-GFP-M mouse brains before and after clearing. (b) The normalized mean fluorescence in (a) $(n=16)$. (c) Confocal imaging of PI-stained mouse brain sections before and after clearing. (d) The normalized mean fluorescence in (c) $(n=9)$. (e) Confocal imaging of blood vessels stained with CD31-A647 antibody before and after clearing. (f) The normalized mean fluorescence in (e) $(n=9)$. All values are presented as the mean \pm SD. Statistical significance in (b), (d), and (f) (n.s., not significant) was assessed by one-way ANOVA followed by the Bonferroni post hoc test.

\section{Discussions and Conclusions}

In this study, we proposed FDISCO+ for robust fluorescence preservation of cleared samples, addressing the issue that FDISCO-cleared samples could not preserve endogenous fluorescent signals well at RT. FDISCO+ shows comparable fluorescence enhancement to FDISCO and achieves long-time fluorescence preservation, enabling 3D visualization of neuronal structures in the mouse brain.

Generally, the environmental temperature of fluorescence microscopy is at RT, which conflicts with the storage condition of FDISCO. Though a short exposure time under RT is acceptable, a long-time imaging process is inevitable when it comes to large-volume imaging at high 


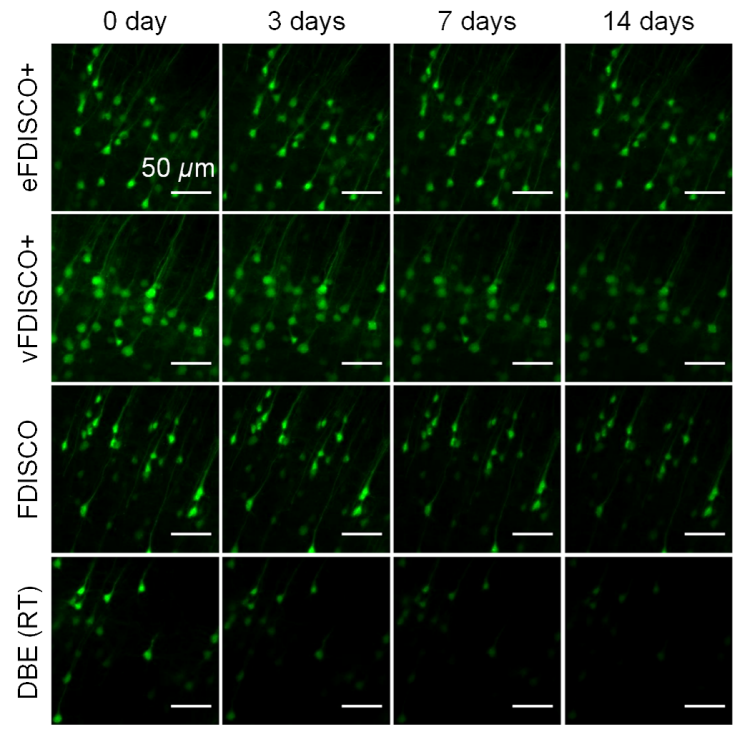

(a)

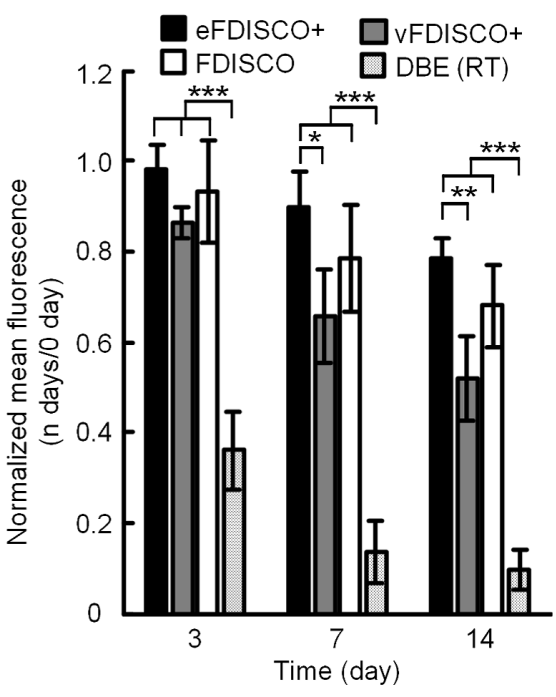

(b)

Fig. 3 FDISCO+ allows long-time preservation of GFP fluorescence at RT. (a) Confocal imaging of cortical neurons in adult Thy1-GFP-M mouse brains cleared after $0,3,7$, and 14 days using FDISCO+ and FDISCO methods. (b) The histogram of normalized mean fluorescence in (a) $(n=6)$. All values are presented as the mean \pm SD. Statistical significance in (b) $\left({ }^{*} p<0.05\right.$; ${ }^{\star *} p<0.01 ;{ }^{* \star} p<0.001$ ) was assessed by one-way ANOVA followed by the Bonferroni post hoc test.

resolution. Some precious samples usually need to be imaged many times. In this case, the fluorescence decrease of FDISCO-cleared samples at RT would impede the 3D imaging and visualization of intact organs or tissues. FDISCO+ eliminates this conflict by realizing the robust fluorescence preservation of cleared samples under RT, making it convenient for longtime storage and long-distance transportation.

As previously mentioned, the peroxides continuously produced in DBE could be the culprit of endogenous fluorescence attenuation. ${ }^{19}$ Hence, we chose two antioxidants that could prevent the peroxide generation in DBE for a long time, involving the metal chelator, EDTP, and the free radical scavenger, vitamin E. Although the EDTP has been used to create an alkaline environment in the CUBIC and PEGASOS, ${ }^{28,34,35}$ it has not been reported to be used as the antioxidant in the clearing methods. The EDTP was often used as a chelating agent, and the chelator has been demonstrated to have antioxidant properties ${ }^{48}$ In this study, we consolidated the antioxidant capacity of the EDTP and firstly used it for inhibiting the generation of peroxides in DBE. The vitamin $\mathrm{E}$ has been used in the uDISCO method to scavenge the peroxides in BABB-D (mixing benzyl alcohol, benzyl benzoate, and diphenyl ether), ${ }^{22}$ but it is unclear whether vitamin E also can scavenge the peroxides in DBE. This work demonstrated the ability to inhibit peroxides by adding low-concentration vitamin $\mathrm{E}$ to DBE. The addition of propyl gallate used in sDISCO method can efficiently preserve EGFP fluorescence signals, but the specimens should also be kept at $\mathrm{LT}\left(4^{\circ} \mathrm{C}\right)$ during all incubation steps and storage.$^{26} \mathrm{It}$ is worth noting that the DBE at LT also did not produce peroxides for a long time, which might be one of the reasons for the long-time preservation of fluorescence in FDISCO. ${ }^{29}$

It has been reported that THF quenches GFP fluorescence by interrupting the hydrogen bonding of protein molecules, and an alkaline $\mathrm{pH}$ and LT environment could reduce the sensitivity of GFP to denaturation by THF. ${ }^{29}$ The basic activated aluminum oxide has been used to remove the peroxides in THF before clearing protocols, and the purchased THF has already been added with $250 \mathrm{ppm}$ BHT (butylated hydroxytoluene) to inhibit the generation of peroxides, so the peroxide in THF is no longer a factor affecting the fluorescent proteins. To preserve the best fluorescence signals, the dehydration procedures of FDISCO+ still adopt the LT and alkaline $\mathrm{pH}$ conditions. 


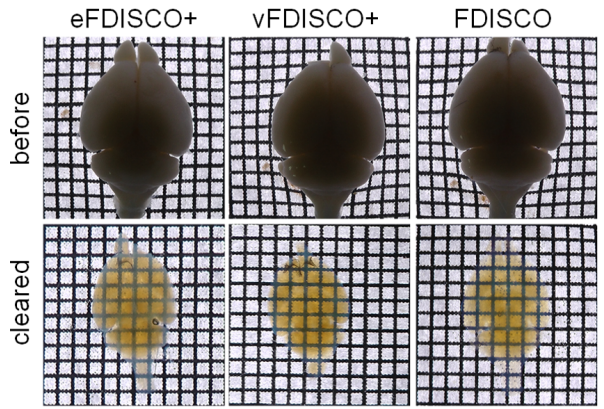

(a)

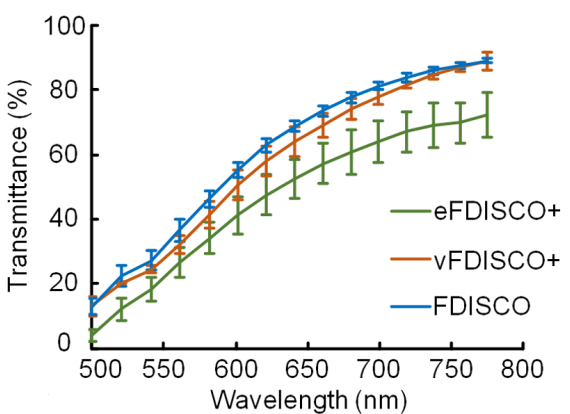

(b)

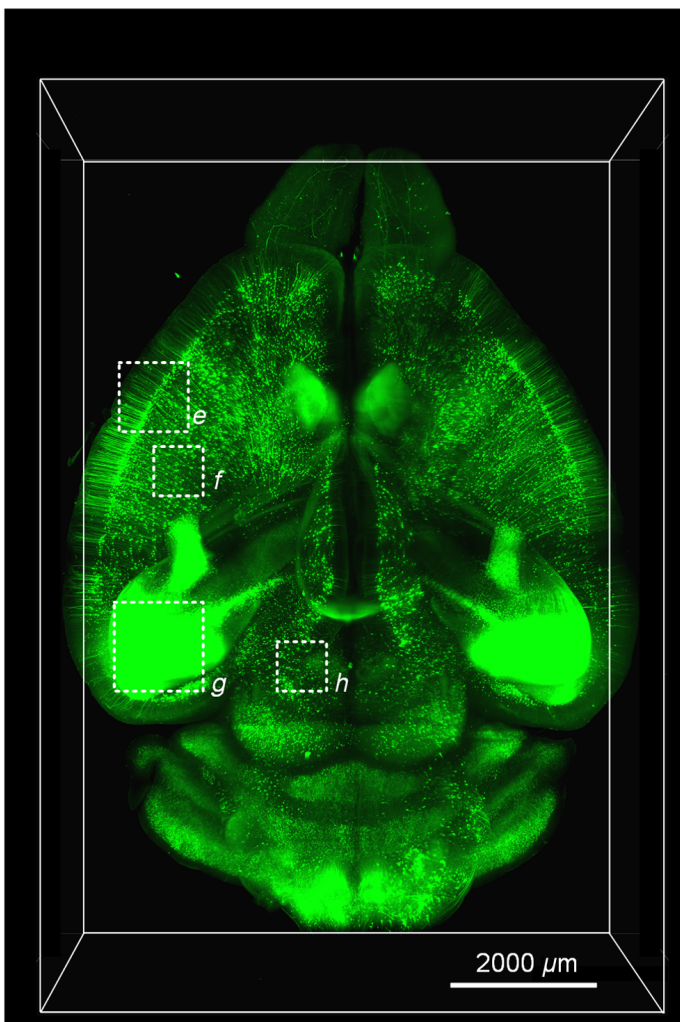

(c)

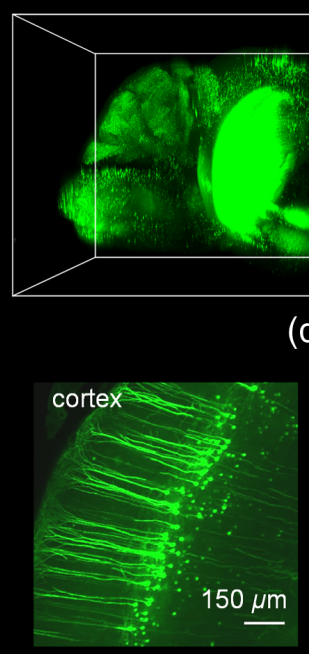

(e)

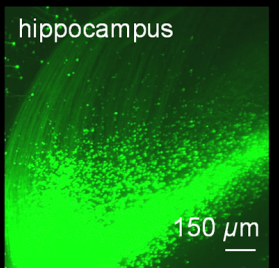

(g) (d)

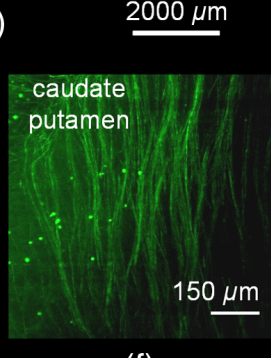

(f)

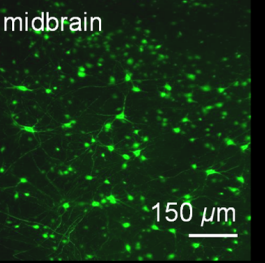

(h)

Fig. 4 FDISCO+ shows good clearing capability and enables visualization of neural structures. (a) Bright-field images of whole brains before and after clearing. (b) The transmittance curves of cleared mouse brains (C57BL/6J mice, 8 weeks old) $(n=3)$. (c), (d) Images of the whole brain (Thy1-GFP-M, 6 weeks old) cleared by vFDISCO+. (e)-(h) The high-magnification images reveal fine structures of different brain areas in (c), including (e) the cortex, (f) caudate putamen, (g) hippocampus, and (h) midbrain. All values are presented as the mean \pm SD (Video 1, MP4, 9821 kB [URL: https://doi.org/10.1117/1.NPh.8.3.035007.1]) and (Video 2, MP4, 7244 kB [URL: https://doi.org/10.1117/1.NPh.8.3.035007.2]).

To meet users' different needs, we developed two strategies of FDISCO+: eFDISCO+ and vFDISCO+. The eFDISCO+ shows better fluorescence preservation than vFDISCO+, whereas vFDISCO+ shows better transparency than eFDISCO+. This might be because the EDTP contains too many hydroxyl groups, and these polar groups influence the osmosis of DBE molecules in the tissue. Users can choose different clearing protocols according to their experimental purposes: for large samples, the vFDISCO+ is an excellent choice; for small samples, the eFDISCO+ may be selected for better fluorescence preservation.

In conclusion, the FDISCO+ method is a simple and convenient clearing method with robust preservation condition, and it is expected to facilitate long-time or repetitive imaging. 


\section{Disclosures}

The authors declare no conflicts of interest.

\section{Acknowledgments}

This study was supported by the National Key Research and Development Program of China (Grant No. 2017YFA0700501), the National Natural Science Foundation of China (Grant Nos. 61860206009, 81870934, 81961138015, and 91749209), and the Innovation Fund of WNLO. The authors would like to thank Peng Fei and Chunyu Fang for their support with light-sheet imaging. The authors also would like to thank the Optical Bioimaging Core Facility of WNLO-HUST for support with data acquisition.

\section{Code, Data, and Materials Availability}

The data used in the manuscript are available upon reasonable request to the corresponding author.

\section{References}

1. D. S. Richardson and J. W. Lichtman, "Clarifying tissue clearing," Cell 162(2), 246-257 (2015).

2. S. W. Oh et al., "A mesoscale connectome of the mouse brain," Nature 508(7495), 207-214 (2014).

3. B. Zingg et al., "Neural networks of the mouse neocortex," Cell 156(5), 1096-1111 (2014).

4. X. F. Yin et al., "Spatial distribution of motor endplates and its adaptive change in skeletal muscle," Theranostics 9(3), 734-746 (2019).

5. J. Zhu et al., "MACS: rapid aqueous clearing system for 3D mapping of intact organs," Adv. Sci. 7(8), 1903185 (2020).

6. J. A. Conchello and J. W. Lichtman, “Optical sectioning microscopy,” Nat. Methods 2(12), 920-931 (2005).

7. J. Mertz, "Optical sectioning microscopy with planar or structured illumination," Nat. Methods 8(10), 811-819 (2011).

8. E. G. Reynaud et al., "Light sheet-based fluorescence microscopy: more dimensions, more photons, and less photodamage," HFSP J. 2(5), 266-275 (2008).

9. J. W. Lichtman and J. A. Conchello, "Fluorescence microscopy," Nat. Methods 2(12), 910 919 (2005).

10. B. Zhang et al., "Multidither coherent optical adaptive technique for deep tissue two-photon microscopy," J. Innov. Opt. Health Sci. 12(4), 1942003 (2019).

11. L. Vannhu, "Optimization method to suppress background for imaging multiple planes," J. Innov. Opt. Health Sci. 12(2), 1950004 (2019).

12. J. Nie et al., "Fast, 3D isotropic imaging of whole mouse brain using multiangle-resolved subvoxel SPIM," Adv. Sci. 7(3), 1901891 (2020).

13. D. Zhu et al., "Recent progress in tissue optical clearing," Laser Photonics Rev. 7(5), 732757 (2013).

14. T. Yu et al., "Optical clearing for multiscale biological tissues," J. Biophotonics 11(2), e201700187 (2018).

15. I. Costantini et al., "In-vivo and ex-vivo optical clearing methods for biological tissues: review," Biomed. Opt. Express 10(10), 5251-5267 (2019).

16. T. Yu et al., "Physical and chemical mechanisms of tissue optical clearing," iScience 24(3), 102178 (2021).

17. H.-U. Dodt et al., "Ultramicroscopy: three-dimensional visualization of neuronal networks in the whole mouse brain," Nat. Methods 4(4), 331-336 (2007).

18. A. Erturk et al., "Three-dimensional imaging of the unsectioned adult spinal cord to assess axon regeneration and glial responses after injury," Nat. Med. 18(1), 166-171 (2012). 
Wan et al.: FDISCO+: a clearing method for robust fluorescence preservation of cleared samples

19. A. Erturk et al., "Three-dimensional imaging of solvent-cleared organs using 3DISCO," Nat. Protoc. 7(11), 1983-1995 (2012).

20. K. Becker et al., "Chemical clearing and dehydration of GFP expressing mouse brains," PLoS One 7(3), e33916 (2012).

21. N. Renier et al., "iDISCO: a simple, rapid method to immunolabel large tissue samples for volume imaging," Cell 159(4), 896-910 (2014).

22. C. C. Pan et al., "Shrinkage-mediated imaging of entire organs and organisms using uDISCO," Nat. Methods 13(10), 859-867 (2016).

23. M. K. Schwarz et al., "Fluorescent-protein stabilization and high-resolution imaging of cleared, intact mouse brains," PLoS One 10(5), e0124650 (2015).

24. A. Klingberg et al., "Fully automated evaluation of total glomerular number and capillary tuft size in nephritic kidneys using lightsheet microscopy," J. Am. Soc. Nephrol. 28(2), 452459 (2017).

25. R. Y. Cai et al., "Panoptic imaging of transparent mice reveals whole-body neuronal projections and skull-meninges connections," Nat. Neurosci. 22(2), 317-327 (2019).

26. C. Hahn et al., "High-resolution imaging of fluorescent whole mouse brains using stabilised organic media (sDISCO)," J. Biophotonics 12(8), e201800368 (2019).

27. Y. Li et al., "Optimization of GFP fluorescence preservation by a modified uDISCO clearing protocol," Front. Neuroanat. 12, 67 (2018).

28. D. Jing et al., "Tissue clearing of both hard and soft tissue organs with the PEGASOS method," Cell Res. 28(8), 803-818 (2018).

29. Y. S. Qi et al., "FDISCO: advanced solvent-based clearing method for imaging whole organs," Sci. Adv. 5(1), eaau8355 (2019).

30. H. Hama et al., "ScaleS: an optical clearing palette for biological imaging," Nat. Neurosci. 18(10), 1518-1529 (2015).

31. M. T. Ke, S. Fujimoto, and T. Imai, "SeeDB: a simple and morphology-preserving optical clearing agent for neuronal circuit reconstruction," Nat. Neurosci. 16(8), 1154-1161 (2013).

32. M. T. Ke et al., "Super-resolution mapping of neuronal circuitry with an index-optimized clearing agent," Cell Rep. 14(11), 2718-2732 (2016).

33. T. Kuwajima et al., "ClearT: a detergent- and solvent-free clearing method for neuronal and non-neuronal tissue," Development 140(6), 1364-1368 (2013).

34. E. A. Susaki et al., "Whole-brain imaging with single-cell resolution using chemical cocktails and computational analysis," Cell 157(3), 726-739 (2014).

35. K. Tainaka et al., "Whole-body imaging with single-cell resolution by tissue decolorization," Cell 159(4), 911-924 (2014).

36. E. A. Susaki et al., "Advanced CUBIC protocols for whole-brain and whole-body clearing and imaging," Nat. Protoc. 10(11), 1709-1727 (2015).

37. W. Li, R. N. Germain, and M. Y. Gerner, "Multiplex, quantitative cellular analysis in large tissue volumes with clearing-enhanced 3D microscopy (Ce3D)," Proc. Natl. Acad. Sci. U. S. A. 114(35), E7321-E7330 (2017).

38. T. Yu et al., "RTF: a rapid and versatile tissue optical clearing method," Sci. Rep. 8, 1964 (2018).

39. K. Chung et al., "Structural and molecular interrogation of intact biological systems," Nature 497(7449), 332 (2013).

40. R. Tomer et al., "Advanced CLARITY for rapid and high-resolution imaging of intact tissues," Nat. Protoc. 9(7), 1682-1697 (2014).

41. B. Yang et al., "Single-cell phenotyping within transparent intact tissue through whole-body clearing," Cell 158(4), 945-958 (2014).

42. J. B. Treweek et al., "Whole-body tissue stabilization and selective extractions via tissuehydrogel hybrids for high-resolution intact circuit mapping and phenotyping," Nat. Protoc. 10(11), 1860-1896 (2015).

43. T. Yu et al., "Elevated-temperature-induced acceleration of PACT clearing process of mouse brain tissue," Sci. Rep. 7, 38848 (2017).

44. P. Wan et al., "Evaluation of seven optical clearing methods in mouse brain," Neurophotonics 5(3), 035007 (2018). 
45. C. Fang et al., "Minutes-timescale 3D isotropic imaging of entire organs at subcellular resolution by content-aware compressed-sensing light-sheet microscopy," Nat. Commun. 12(1), 107 (2021).

46. T. Yu et al., "Rapid and prodium iodide-compatible optical clearing method for brain tissue based on sugar/sugar-alcohol," J. Biomed. Opt. 21(8), 081203 (2016).

47. N. Otsu, "A threshold selection method from gray-level histograms," IEEE Trans. Syst. Man Cybern. Syst. 9, 62-66 (1979).

48. A. Balcerczyk, K. Sowa, and G. Bartou, "Metal chelators react also with reactive oxygen and nitrogen species," Biochem. Biophys. Res. Commun. 352(2), 522-525 (2007).

Peng Wan received his bachelor's degree in biomedical engineering from Huazhong University of Science and Technology (HUST) in 2017. He is currently working toward his PhD in biomedical photonics at Wuhan National Laboratory for Optoelectronics of HUST. His main research interest includes in vitro tissue optical clearing methods and applications in diabetic models.

Yusha Li received her bachelor's degree in biomedical engineering from China Medical University in 2015 and her MS degree in biomedical engineering from Wuhan National Laboratory for Optoelectronics of HUST in 2018, where she is pursuing her PhD. Her main research interests are in vitro tissue optical clearing and three-dimensional immunofluorescence staining.

Jingtan Zhu received his PhD in optical engineering from HUST in 2020. He is currently working as a postdoctoral fellow at Wuhan National Laboratory for Optoelectronics of HUST. His research interests include in vitro tissue optical clearing methods and fluorescence imaging of various tissues and organs.

Jianyi Xu received his bachelor's degree in biotechnology from Huazhong Agricultural University in 2015. He is currently pursuing his $\mathrm{PhD}$ in biomedical engineering at Wuhan National Laboratory for Optoelectronics of HUST. His research interests include in vitro tissue optical clearing methods and fluorescence imaging of the mouse peripheral nervous system.

Xiaomei Liu received her bachelor's degree in biomedical engineering from Tianjin Medical University in 2020. She is currently working toward her PhD in biomedical engineering at Wuhan National Laboratory for Optoelectronics of HUST. Her research interest is in vitro tissue optical clearing methods for whole-organ angiography.

Tingting Yu received her $\mathrm{PhD}$ in biomedical photonics from HUST in 2015. She is currently an associate researcher at Wuhan National Laboratory for Optoelectronics of HUST. Her research activity is focused on tissue optical clearing method and neuroimaging with various optical imaging systems, including light-sheet microscopy, and single/two-photon microscopy.

Dan Zhu received her PhD in physical electronics from HUST in 2001. She is currently a full professor of Wuhan National Laboratory for Optoelectronics and a fellow of SPIE. She has engaged in the study of tissue optical imaging, including new theories, new technologies, and methods. Recently, her research interest is focused on tissue optical clearing of skin, skull, neuronal tissues, and other organs. 